\title{
Hepatic Failure Due to Hepatitis E Virus Infection in a Patient with Necrotic Hepatocellular Carcinoma
}

\author{
Ji Hye Kim, Young Seok Doh, Ji Woong Jang, Min Seok Kang, Nak Min Kim, Sae Hee Kim, Il Hyun Baek, Sung Hee Jung \\ Division of Gastroenterology, Department of Internal Medicine, Eulji University Hospital, Eulji University College of Medicine, Daejeon, Korea
}

Received Dec. 21, 2018

Revised Dec. 31, 2018

Accepted Dec. 31, 2018
In patients with hepatocellular carcinoma (HCC) or liver cirrhosis (LC) accompanied by hepatitis $E$ virus (HEV) infection, hepatic failure often leads to debility. Here, we report about a 63-year-old man with alcoholic LC who was referred to our hospital with jaundice and abdominal distension 10 days earlier. Abdominal computed tomography showed necrotic HCC accompanied by left lobe shrinkage without tumor progression. Laboratory and imaging findings revealed no acute infection focus. The patient reported no herbal medicine or alcohol consumption, and there was no evidence of acute viral hepatitis. One month later, HEV immunoglobulin M positivity was confirmed, and deterioration of liver function due to HEV infection was suspected. The patient often ate raw oysters and sashimi, as well as boar meat, which is a well-known risk food for HEV infection. His umbilical hernia deteriorated due to tense ascites and infection by skin abrasion. The patient progressed to hepatorenal syndrome and eventually died. Liver function preservation is important when treating HCC patients. Therefore, clinicians should pay more attention to the prevention of HEV and others causes of direct liver injury. (J Liver Cancer 2019;19:55-58)

Keywords: Hepatocellular carcinoma; Regression; Hepatitis E virus; Liver failure

\section{INTRODUCTION}

In patients with hepatocellular carcinoma (HCC) or liver cirrhosis (LC), hepatitis E virus (HEV) infection often leads to debility through rapid deteriotation of hepatic function. Although well-known risk factors, such as the consumption of alcohol and herbal medicines, are emphasized, preventive education for HEV infection is not sufficiently practiced.

\section{Corresponding author : Young Seok Doh}

Division of Gastroenterology, Department of Internal Medicine, Eulji University Hospital, Eulji University College of Medicine, 95 Dunsanseo-ro, Seo-Gu, Daejeon 35233, Korea

Tel. +82-42-611-3791, Fax. +82-42-259-1111

E-mail; elimier@gmail.com

https://orcid.org/0000-0003-2682-2726
Here, we report a 63-year-old man with alcoholic LC and necrotic HCC who died of hepatic insufficiency due to HEV infection.

\section{CASE REPORT}

\section{Clinical findings}

A 63-year-old man was referred to Eulji University Hospital for severe jaundice and abdominal distension on 5 May 2017. After a diagnosis of alcoholic LC and HCC on 21 November 2016, he refused treatment for HCC. Instead, he abstained from alcohol consumption and maintained a good general condition, without ascites, jaundice, or encephalopathy. The HCC did not progress after the development of 
spontaneous necrotic change.

The patient presented with yellowish skin color and was thin except for the abdominal distension, which had developed 10 days earlier. There was no history of herbal medications. His only recent medicine before hospitalization was amoxicillin and clavulanic acid for one week for mild flu-like symptoms.

\section{Laboratory and imaging findings}

He showed high bilirubin (total bilirubin [T-bilirubin]: $33.1 \mathrm{mg} / \mathrm{dL}$, direct bilirubin [D-bilirubin]: $24.5 \mathrm{mg} / \mathrm{dL}$ ) compared to his previous value (T-bilirubin: $1.27 \mathrm{mg} / \mathrm{dL}$ ) and normal alanine aminotransferase (ALT) and elevated aspartate aminotransferase (AST) levels (ALT: 27 IU/L, AST: 48 IU/L). The other test results were as follows: gamma-glutamyltransferase, 49 IU/L; elevated alkaline phosphatase activity, 149 $\mathrm{IU} / \mathrm{L}$; albumin, $3.1 \mathrm{~g} / \mathrm{L}$; and international normalized ratio (INR), 1.39. His blood hemoglobin (Hb) was $11.4 \mathrm{~g} / \mathrm{dL}$; the white blood cell (WBC) count was $6 \times 10^{3} / \mu \mathrm{L}$ and his platelet count (PLT) was low at $34 \times 10^{3} / \mu \mathrm{L}$. The $\mathrm{C}$-reactive protein concentration was $1.62 \mathrm{mg} / \mathrm{dL}$.

Abdominal computed tomography (CT) showed necrotic HCC accompanied by left lobe shrinkage and partial thrombosis involving the main, left, and right portal veins, as well as the superior mesenteric vein, without biliary obstruction
(Fig. 1). According to the CT imaging findings, the possibility of jaundice due to HCC progression was low. To address the acute exacerbation of hepatic function, serologic tests, chest X-ray, urine analysis, and paracentesis (ascites analysis: $\mathrm{WBC}, 96 / \mu \mathrm{L}$ red blood cells, $650 / \mu \mathrm{L}$ neutrophils, $8 \%$; lymphocytes, 17\%; other cells, 75\%; and no growth in culture study) were performed, but there was no infection focus and the results of viral markers were all negative (anti-hepatitis A virus [HAV] immunoglobulin [Ig] M; hepatitis B surface antigen/anti-hepatitis B surface antibody; anti-hepatitis B core antigen IgM; anti-hepatitis C virus; and serological results for acute Epstein-Barr and cytomegaloviral infection). However, we could not obtain HEV IgM and HEV IgG results because the process required 3 weeks.

\section{Diagnosis and treatment progress}

The patient was administered antibiotics for a potential hidden infection and was conservatively managed with diuretics, dextrose, and albumin infusion. In addition, we checked the levels of alpha-fetoprotein $(3.2 \mathrm{ng} / \mathrm{mL})$ and proteins induced by vitamin $\mathrm{K}$ absence or antagonist-II (PIVKA-II, $13 \mathrm{mAU} / \mathrm{mL}$ ). After two weeks, he was discharged; his bilirubin had decreased to $19.9 \mathrm{mg} / \mathrm{dL}$ (T-bilirubin: $19.9 \mathrm{mg} / \mathrm{dL}$, D-bilirubin: $13.0 \mathrm{mg} / \mathrm{dL}$, ALT: $27 \mathrm{IU} / \mathrm{L}$, AST: 48 IU/L, INR: 1.39, PLT: $\left.34 \times 10^{3} / \mu \mathrm{L}\right)$, and the ascites was controlled with

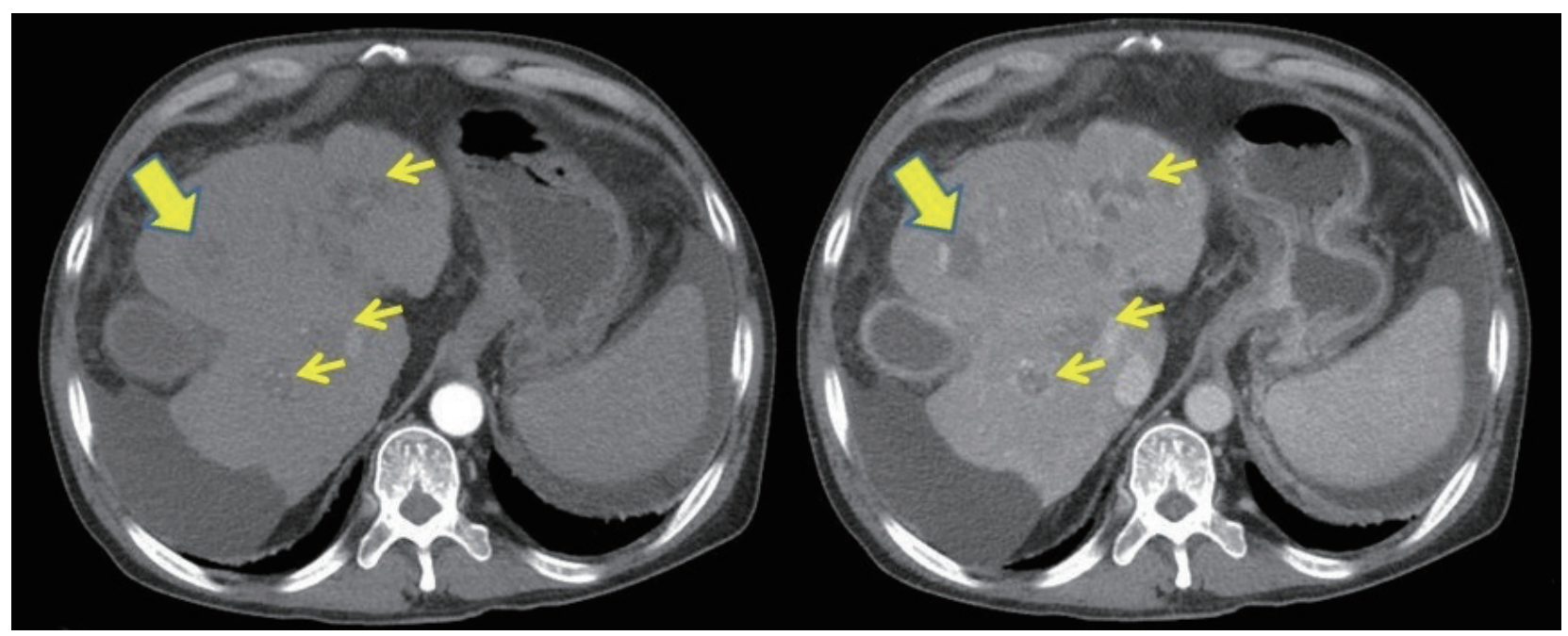

Figure 1. Abdominal computed tomography showing a $2.4 \mathrm{~cm}$, round, low-attenuating mass in segment VIII (thick arrows) as well as main, left, and right portal vein thrombus (edged arrows). 
diuretics.

Two weeks later, the patient visited an outpatient clinic, reporting a recovered general condition but with ascites. His bilirubin remained high compared to the previous, healthy level. We finally confirmed HEV IgM positivity and retrospectively diagnosed the patient with an aggravation of hepatic insufficiency due to HEV infection. ${ }^{1}$ This finding was well-correlated with his exposure history to hazardous foods, such as the oysters and sashimi that he often enjoyed; we also confirmed that he had eaten boar meat twice three weeks before his first admission.

The patient was admitted to the hospital with recurrent jaundice, and showed a steady clinical course, with $\mathrm{Hb}$ of $10.9 \mathrm{~g} / \mathrm{dL}$, PLT of $19.1 \times 10^{3} / \mu \mathrm{L}$, albumin of $4.0 \mathrm{mg} / \mathrm{dL}$, protein of $6.7 \mathrm{mg} / \mathrm{dL}$, AST of $36 \mathrm{IU} / \mathrm{L}$, ALT of $22 \mathrm{IU} / \mathrm{L}$, and Tbilirubin of $24.3 \mathrm{mg} / \mathrm{dL}$, without other symptoms and interventions, except for intermittent therapeutic paracentesis.

One month later, the patient was admitted again with progressed jaundice and weakness. Erosion and swelling were observed in the vicinity of an umbilical hernia and were due to skin abrasion from his clothing. The patient was discharged slightly recovered after antibiotic treatment.

However, he was readmitted to the hospital for oozing bleeding from the umbilical hernia. Although hemostasis was achieved through manual compression and correction of coagulopathy, the inflammation persisted. Eventually, the patient progressed to hepatorenal syndrome and died.

\section{DISCUSSION}

While the patient was diagnosed with alcoholic cirrhosis and liver cancer, the progression of liver cancer was not very rapid; the tumor showed focal necrosis, and it was possible to observe clinical progress without specific treatment for HCC according to his intent. However, his liver function deteriorated due to the recurrence of acute hepatic injury including HEV infection that resulted in massive ascites, infection, bleeding of his umbilical hernia, and finally in death. The present case had two characteristic features. The progression of HCC was not rapid and did not affect the clinical course of the patient, showing focal necrosis and lobar shrinkage. Meanwhile, hepatocellular injury caused by HEV infection was the main cause of the disease aggravation, leading to death.

Spontaneous regression or necrosis is a rare phenomenon believed to be induced mainly by ischemia and systemic inflammation such as bacterial or viral infection. ${ }^{2,3}$ In this case, portal vein and superior mesenteric vein thrombosis were obviously observed, and the mean arterial pressure was slightly less than $75 \mathrm{mmHg}$ at admission. Therefore, it was possible that the HCC necrosis was caused by ischemia. ${ }^{4}$ In addition, since the timing of the infection was unclear and there was no severe inflammation at admission, it was not clear if the HEV infection affected the tumor necrosis.

In the present case, HEV was the only major infectious source identified to explain the liver damage. No other causes, such as bacterial infections, bleeding, azotemia, toxic liver damage, or alcohol, could explain the acute hepatic dysfunction. Ultimately, this is a case report of an HCC patient who died without tumor progression due to deteriorating clinical features after HEV infection, a viral infection rarely described in Korean case reports.

We could not prevent the liver damage due to the retrospective confirmation of the HEV infection and his initial diagnosis in another hospital. There may be a high risk of similar cases in Korea because of its food culture that often incorporates sashimi, oysters, and occasional boar meat. ${ }^{1,5-7}$ Therefore, prospective prevention of similar cases is necessary. ${ }^{8}$ Although there are few case reports, HEV is thought to be prevalent in offshore marine life in Korea ${ }^{7}$ and may be a direct cause of liver damage in patients with liver cancer and LC. ${ }^{9}$ However, during treatment of HCC, patients are not usually tested for HEV infection, even if the cause of acute hepatic insufficiency is unspecified. Furthermore, education on the prevention of HEV infection tends to be insufficient. Therefore, all clinicians should pay increased attention to infection prevention.

In addition, the prevalence of hepatitis $\mathrm{A}$ and waterborne infections such as HEV has recently increased in Korea. ${ }^{10}$ The number of HCC patients without protective HAV IgG may also have increased. ${ }^{11}$ Preventive education regarding hygiene should be provided and immunization status should be 
checked carefully.

\section{Conflicts of Interest}

The authors have no conflicts to disclose.

\section{REFERENCES}

1. Jeong SH. Current status of hepatitis E virus infection in Korea. Gut Liver 2011;5:427-431.

2. Blondon H, Fritsch L, Cherqui D. Two cases of spontaneous regression of multicentric hepatocellular carcinoma after intraperitoneal rupture: possible role of immune mechanisms. Eur J Gastroenterol Hepatol 2004;16:1355-1359.

3. Cole WH. Efforts to explain spontaneous regression of cancer. J Surg Oncol 1981;17:201-209.

4. Randolph AC, Tharalson EM, Gilani N. Spontaneous regression of hepatocellular carcinoma is possible and might have implications for future therapies. Eur J Gastroenterol Hepatol 2008;20:804-809.

5. Park WJ, Park BJ, Ahn HS, Lee JB, Park SY, Song CS, et al. Hepatitis
E virus as an emerging zoonotic pathogen. J Vet Sci 2016;17:1-11.

6. Song YJ, Park WJ, Park BJ, Lee JB, Park SY, Song CS, et al. Hepatitis E virus infections in humans and animals. Clin Exp Vaccine Res 2014;3:29-36.

7. Shin HK, Yoon JD, Yoo JC, Kim MB, Kim KS, Suh SD, et al. Prevalence of hepatitis $E$ virus antibody on residents of seashore town in Korea. J Korean Soc Virol 1993;23:215-222.

8. Lim JW, Park CS, Ahn JM, Yu MH, Kim TS, Lim YS, et al. Nine cases of sporadic acute hepatitis $\mathrm{E}$ in Korea. Korean J Hepatol 2006;12:230-236.

9. Barragué $H$, Condat $B$, Petitdidier N, Champagne E, Renou C, Izopet J, et al. Chronic hepatitis E virus infection in a cirrhotic patient. Medicine 2016;96:e7915.

10. Moon S, Han JH, Bae GR, Cho E, Kim B. Hepatitis A in Korea from 2011 to 2013: current epidemiologic status and regional distribution. J Korean Med Sci 2016;31:67-72.

11. Choi JS, Ko JW, Park S. Factors associated with hepatitis A preventative behaviors among university students. Korean J Adult Nurs 2015;27:127-134. 